\title{
Toxoplasma gondii oocyst-driven infection in pigs, chickens and humans in northeastern China
}

\author{
Xiao-Yi Liu', Ze-Dong Wang ${ }^{1,2}$, Saeed El-Ashram ${ }^{1}$ and Quan Liu' ${ }^{1,2^{*}}$
}

\begin{abstract}
Background: Toxoplasma gondii, an intracellular apicomplexan protozoan parasite, can infect almost all warmblooded animals. The aim of the present study was to investigate T. gondii oocyst-driven infection in pigs, chickens and humans in Jilin province, northeastern China.

Results: The serum samples of pigs, chickens and humans were sampled and tested by indirect enzyme-linked immunosorbent assays (ELISAs) using dense granule antigen GRA7, oocyst-specific protein OWP8, and sporozoitespecific protein CCP5A, respectively. Results showed a prevalence of $16.7 \%$ by GRA7-ELISA, and $12.2 \%$ by OWP8and CCP5A-ELISA in pigs; $10.4 \%$ by GRA7-ELISA, $13.5 \%$ by OWP8-ELISA, and $9.4 \%$ by CCp5A-ELISA in chickens; and $14.2 \%$ by GRA7-ELISA, 3.6\% by OWP8-ELISA, and 3.0\% by CCP5A-ELISA in humans. No significant differences were observed between T. gondii seroprevalence in pigs and chickens among the three antigens-based ELISAs $(P>0.05)$. However, there were significant differences between T. gondii seroprevalence rates in humans $(P<0.05)$. These findings demonstrated a low prevalence of $T$. gondii oocyst-driven infection in humans, a medium prevalence in pigs, and a high prevalence in chickens.
\end{abstract}

Conclusions: The present study demonstrated that different oocyst-driven infection rates in different animal species, which would help to design effective strategies to prevent $T$. gondii transmission. To our knowledge, this is the first study to differentiate T. gondii infective forms in pigs, chickens and humans in China.

Keywords: Toxoplasma gondii, Oocyst, Sporozoite, GRA7, OWP8, CCp5A, ELISA

\section{Background}

Toxoplasma gondii, an intracellular apicomplexan protozoan parasite, can infect nearly all warm-blooded animals, including humans and birds [1]. In human, $T$. gondii infection can be life-threatening for congenitally infected infants and immunocompromised patients [2]. The definitive hosts of $T$. gondii are members of the Felidae family, and the intermediate hosts include almost all warm-blooded animals. The three parasite stages, including the tachyzoites, the bradyzoites in the tissue cysts, and the sporozoites in the oocysts, are able to infect the hosts [3]. The oral route is considered the main form of postnatal transmission of toxoplasmosis in

\footnotetext{
* Correspondence: liuquan1973@hotmail.com

${ }^{1}$ College of Life Science and Engineering, Foshan University, Foshan, Guangdong Province, China

${ }^{2}$ Military Veterinary Institute Academy of Military Medical Sciences, Changchun, Jilin Province, China
}

humans and animals, which may be obtained by ingestion of infective oocysts in contaminated food and water, or by ingestion of raw/undercooked meat containing tissue cysts $[2,3]$.

The $T$. gondii oocysts are highly resistant to freezing or disinfectants, and sporulated oocysts can survive in moist soil for months or even years, and also can be mechanically transferred from one host to the other by invertebrates [2]. Therefore, an environmental contamination with $T$. gondii oocysts is also considered as a potential source and a risk factor for warm-blooded animals, including humans. Due to the great medical importance of toxoplasmosis, numerous epidemiological surveys have been previously conducted in humans and other animals worldwide [4-10]. However, few studies of them have identified the infection sources of $T$. gondii.

(c) The Author(s). 2019 Open Access This article is distributed under the terms of the Creative Commons Attribution 4.0 International License (http://creativecommons.org/licenses/by/4.0/), which permits unrestricted use, distribution, and reproduction in any medium, provided you give appropriate credit to the original author(s) and the source, provide a link to the Creative Commons license, and indicate if changes were made. The Creative Commons Public Domain Dedication waiver (http://creativecommons.org/publicdomain/zero/1.0/) applies to the data made available in this article, unless otherwise stated. 
Several oocyst/sporozoite-specific proteins have been identified as serological markers for oocyst-driven $T$. gondii infection. For example, the oocyst wall protein 8 (OWP8) has been confirmed to be oocyst-specific, without cross-reactivity to bradyzoite cyst wall or tachyzoite antibodies [11]. In addition, the sporozoite-specific protein, embryogenesis- related protein (ERP), has been used to differentiate oocyst- versus tissue cyst-induced infection in humans and animals [12]. Furthermore, the Limulus factor C-Coch-5b2-Lgl1 (LCCL) related protein, $\mathrm{CCp} 5 \mathrm{~A}$, which is a common feature of various secreted proteins among apicomplexan parasites, expressed only in the oocyst stage of $T$. gondii, and can tell T. gondii oocyst infection from tissue cyst infection in vivo [13]. Another T. gondii marker, the dense granule antigen protein 7 (GRA7), an important secretory protein, expressed by $T$. gondii tachyzoites and bradyzoites. GRA7 locates on the surface and cytoplasmic matrix of host cells. Studies have shown that the recombinant GRA7 can perform well in the diagnostic of T. gondii infection in animals, including chicken, dog, cat, cattle, and human, but cannot differentiate oocyst- versus tissue cyst- induced T. gondii infection [14-19].

Identification of $T$. gondii infective forms can help to design effective strategies to control parasite transmission and to prevent severe complications, mainly in immunocompromised people and pregnant women. In this study, serologic differentiation of $T$. gondii infective stages in human, pig and chicken infection was conducted in Jilin province, northeastern China, using GRA7 and oocyst/ sporozoite-specific proteins OWP8 and CCp5A.

\section{Results}

\section{Cloning and expression of OWP8 and CCp5A}

The OWP8-encoding gene was cloned into the prokaryotic vector pET30, and expressed in E. coli BL21 (DE3).
The protein was purified by Ni-NTA affinity chromatography, showing a histidine fusion protein of approximately $65 \mathrm{kDa}$ (Fig. 1a). The immunoreactivity of the expressed protein was confirmed by Western blot using the mice serum anti-His tag IgG antibodies, showing a specific signal at approximately $75 \mathrm{kDa}$ (Fig. 1b).

The CCp5A, a LCCL-related protein, contains three important domains (468 bp) that was used to construct recombinant expression vector $\mathrm{pET} 30-\mathrm{CC} 5 \mathrm{~A}$. The protein was expressed as a histidine fusion protein of approximately $17 \mathrm{kDa}$. After analyzed with coomassie brilliant blue staining SDS-PAGE (Fig. 1c), the recombinant protein was also confirmed by Western blot using anti-His tag IgG antibodies (Fig. 1d).

\section{Serologic differentiation of $T$. gondii infective stages in pigs}

To figure out whether OWP8 and CCp5A can serve as the protein markers of differentiating $T$. gondii infective stages, $15 \mathrm{~T}$. gondii tachyzoite positive and 10 negative porcine serum samples were detected by GRA7-, OWP8-, and CCp5A-ELISAs, respectively. As expected, all the samples could be correctly distinguished by GRA7, with a mean ELISA index (EI, EI=OD of each sample/cut off value) of 3.77 and 0.56 for GRA7-positive and negative samples, respectively. OWP8 and CCp5A showed no reactivity for all serum samples, except for one sample had a mean EI of 0.66 and 0.52 for OWP8positive and negative samples, and one sample with a mean EI of 0.44 and 0.36 for CCp5A-positive and negative samples, respectively (Fig. 2a). On Western blot, GRA7 presented reactivity for all the positive samples but none of the samples showed reactivity for OWP8 and CCp5A (Fig. 2b).

As shown in Table 1, of the 90 serum samples of freerange pigs, which were tested by GRA7-, OWP8-, and
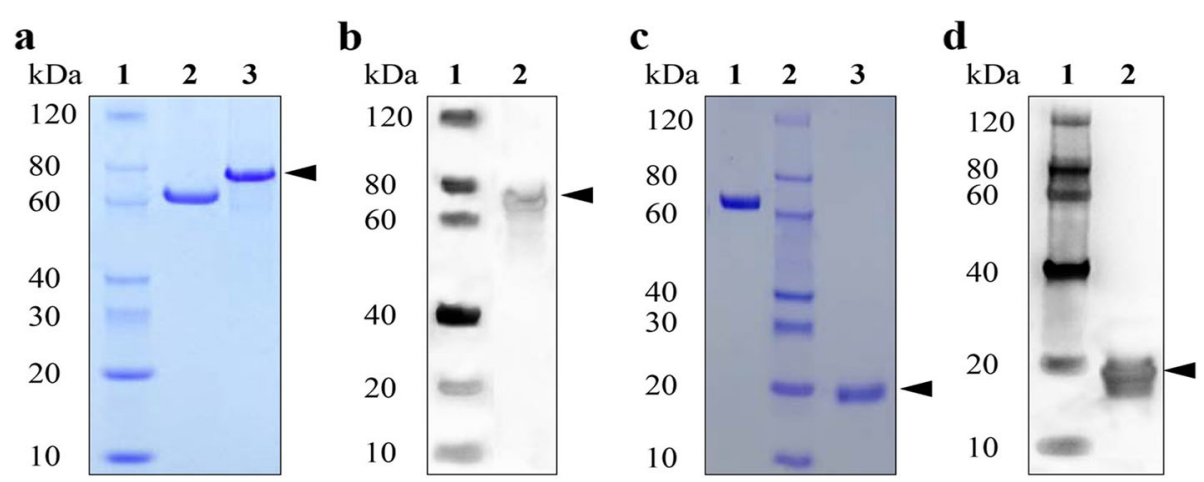

Fig. 1 Expression of recombinant OWP8 and CCP5A of T. gondii in E. coli. a SDS-PAGE analysis of the purified OWP8. Lane 1: Protein molecular weight marker; Lane 2: BSA $(2 \mu \mathrm{g})$; Lane 3: The purified OWP8 by Ni-NTA affinity chromatography $(2 \mu \mathrm{g})$. $\mathbf{b}$ Western blot analysis of the recombinant OWP8. Lane 1: Molecular protein marker; Lane 2: The expressed OWP8. c The purified CCP5A analyzed by SDS-PAGE. Lane 1: BSA; Lane 2: Molecular protein marker; Lane 3: The purified CCp5A. d Western blot analysis of the recombinant CCp5A. Lane 1: Protein molecular weight marker; Lane 2: The expressed CCP5A; The arrows indicate the target protein 

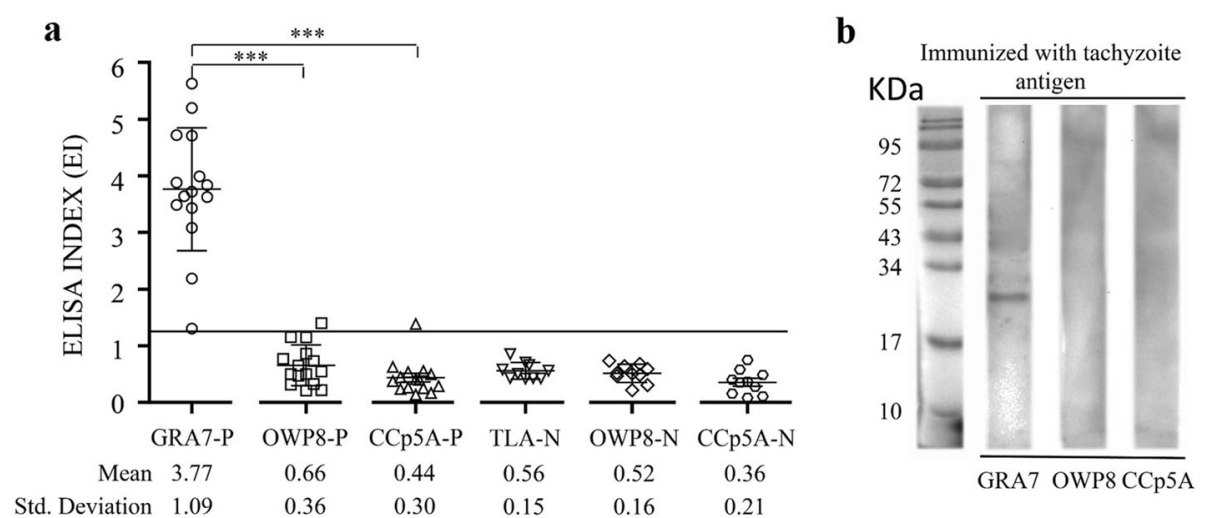

Fig. 2 Detection of T. gondii tachyzoite positive and negative porcine serum samples by ELISA and Western blot using different antigens. a The porcine positive and negative serum samples were tested by GRA7-, OWP8-, and CCP5A-ELISAs. The dashed line indicates the cut off of the reactions. GRA7-P, OWP8-P and CCP5A-P represented the porcine positive samples for T. gondii tachyzoite infection, while GRA7-N, OWP8-N and CCp5A-N represented the porcine negative serum samples for $T$. gondii infection. $\mathbf{b}$ Western blot using serum samples from pigs experimentally infected with tachyzoites. The molecular weight markers are shown at the left side

CCp5A-ELISA, respectively, there were 15 positive and 75 negative samples by GRA7- ELISA, 11 positive and 79 negative samples by both OWP8-, and CCp5AELISAs. There was no significant difference found in mean EI values (Fig. 3a). The following seroprevalence results were obtained: $16.7 \%(95 \%[\mathrm{CI}], 9.0$ to 24.4$)$ by GRA7- ELISA, and 12.2\% (95\% [CI], 5.5 to 19.0) by both OWP8- and CCp5A -ELISA (Fig. 3b). Ten positive samples on both OWP8- and CCp5A-ELISA were detected among those 15 GRA7-ELISA positive samples, suggesting that $T$. gondii oocyst-driven infection rate in T. gondii positive porcine serum samples was $66.7 \%$ (10/15) (Table 1).

\section{Serologic differentiation of $T$. gondii infective stages in chickens}

To determine sources of $T$. gondii infection in chickens, a total of 96 serum samples from free-range chickens were tested by OWP8- and CCp5A-ELISA, and compared with GRA7-ELISA. There were 10 GRA7- ELISA positive samples, while there were 13 and 9 positives in OWP8-, and CCp5A-ELISAs, respectively (Table 1).

The IgY antibodies levels were expressed by ELISA index (EI), showing EI means of 0.64 for GRA7, 0.69 for OWP8 and 0.56 for CCp5A (Fig. 4a). The following positivity rates were obtained: $10.4 \%$ (95\% CI, 4.3 to 16.5) for GRA7, $13.5 \%$ (95\% CI, 6.7 to 20.4) for OWP8, and $9.4 \%$ (95\% CI, 3.5 to 15.2) for CCp5A (Fig. 4b). There were no significant differences of the ELISAs and prevalence of $T$. gondii among GRA7-, OWP8-, and CCp5A-ELISAs $(P>0.05)$. Such results indicated a high $T$. gondii oocyst-driven infection rate in $T$. gondii positive chicken serum samples of $100 \%(10 / 10)$ by OWP8ELISA and $80 \%(8 / 10)$ by CCp5A-ELISA, respectively (Table 1).

\section{Serologic differentiation of $T$. gondii infective stages in humans}

To distinguish sources of $T$. gondii infection in humans, human serum samples were tested for the recombinant proteins. Of the 169 samples tested, 24 samples were positive for GRA7-ELISA, 6 for OWP8-ELISA, and 5 for CCp5A-ELISA (Table 1). Testing of IgG antibodies by ELISA showed the following EI means: 0.93 for GRA7, 0.57 for OWP8, and 0.54 for CCp5A, respectively (Fig. 5a). The following seroprevalence rates were recorded: $14.2 \%$ (95\% [CI], 8.1 to 20.4) for GRA7, 3.6\% (95\% [CI], 1.4 to 8.0 ) for OWP8, and 3.0\% (95\% [CI], 1.0 to 7.2) for CCp5A (Fig. 5b). There were significant differences between ELISA indices and seroprevalences of T. gondii by GRA7-ELISA and OWP8-, CCp5A- ELISAs, respectively $(P<0.05)$. Among the GRA7-ELISA positive human serum samples, $25.0 \%(6 / 24)$ and $20.8 \%(5 / 24)$ were detected as OWP8-CCP5A-ELISAs positive,

Table 1 Detection results of T. gondii antibodies by ELISA based on different antigens

\begin{tabular}{lllll}
\hline Species & No. tested & No. (\%) positive for GRA7 & No. (\%) positive for OWP8 & No. (\%) positive for CCp5A \\
\hline Pigs & 90 & $15(16.7)$ & $11(12.2)$ & $11(12.2)$ \\
Chickens & 96 & $10(10.4)$ & $13(13.5$ & $9(9.4)$ \\
Humans & 169 & $24(14.2)$ & $6(3.6)$ & $5(3.0)$
\end{tabular}



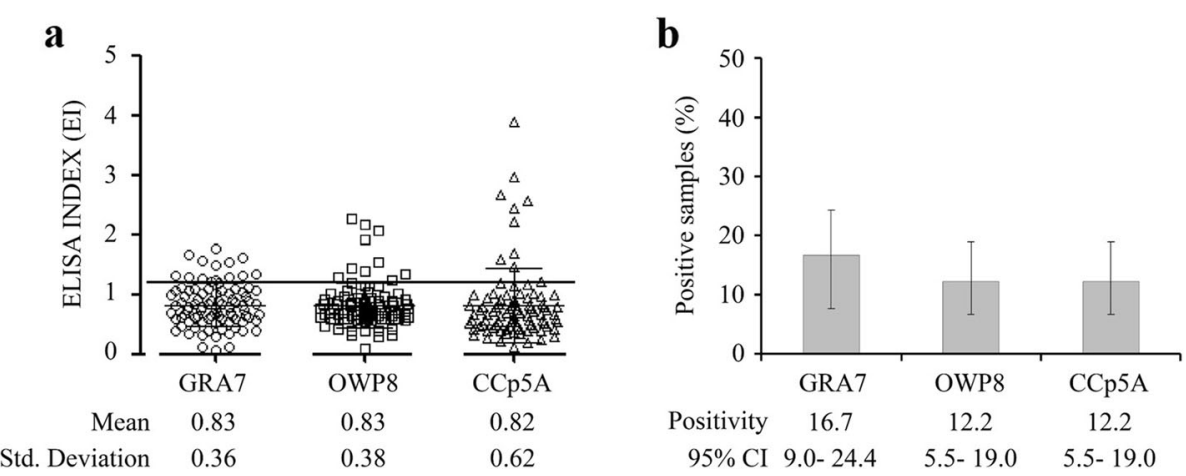

Fig. 3 Detection of anti-T. gondii antibodies in serum samples of free-range pigs using different antigens. a The porcine serum samples were tested by GRA7-, OWP8-, and CCP5A- ELISA, The dashed line indicates the cut off of the reactions. b Positivity was evaluated using GRA7-, OWP8-, and CCP5A-ELISAs in serum samples from free range pigs $(n=90)$

respectively, which suggested that a low $T$. gondii oocyst-driven infection rate in human beings (Table 1).

\section{Discussions}

Previous studies have demonstrated that the oocysts/ sporozoite-specific proteins OWP8 and CCp5A show specific reactivity for oocyst-infected animals, without reactivity for cysts- or tachyzoites-infected animals, while GRA7 is an important secretory protein expressed by $T$. gondii tachyzoites and bradyzoites, and is a sero-diagnostic marker for T. gondii infection in kinds of animals $[11,13-15,17,18]$. In this study, T. gondii infection was firstly screened in different animals using GRA7-ELISA. Subsequently, the positive samples were tested by OWP8- and CCp5AELISAs, respectively. The results showed different oocyst-driven infection rates between 3.0 and $16.7 \%$, ranging from a low prevalence in humans, a medium prevalence in pigs, to a high prevalence in chickens. However, further studies should be conducted on other animal species or other regions involving larger sample sizes. To our knowledge, this is the first study to differentiate infective stages of $T$. gondii in pigs, chickens and humans in China.

The specificity of oocysts/sporozoites specific proteins OWP8-, and CCp5A were also confirmed using pig serum samples immunized with tachyzoites, demonstrating that GRA7-ELISA showed a perfect detection on both positive and negative samples, while OWP8 and CCp5A showed no reactivity for all serum samples. Such results confirmed that OWP8 and CCp5A cannot detect antibodies preferentially in sera from pigs infected by $T$. gondii tachyzoite, which were further verified by Western blot.

Our study showed different oocyst-driven infection rates in different hosts of $T$. gondii, which are associated with their feeding habits or lifestyles. Consumption of raw or undercooked meat containing $T$. gondii tissue cysts is one of the main transmission routes in humans, and other infection routes also include consuming food or drink contaminated with oocysts or by accidentally ingesting oocysts from the environment, which are consistent with our findings that approximately $25 \%$ oocyst-driven infections and
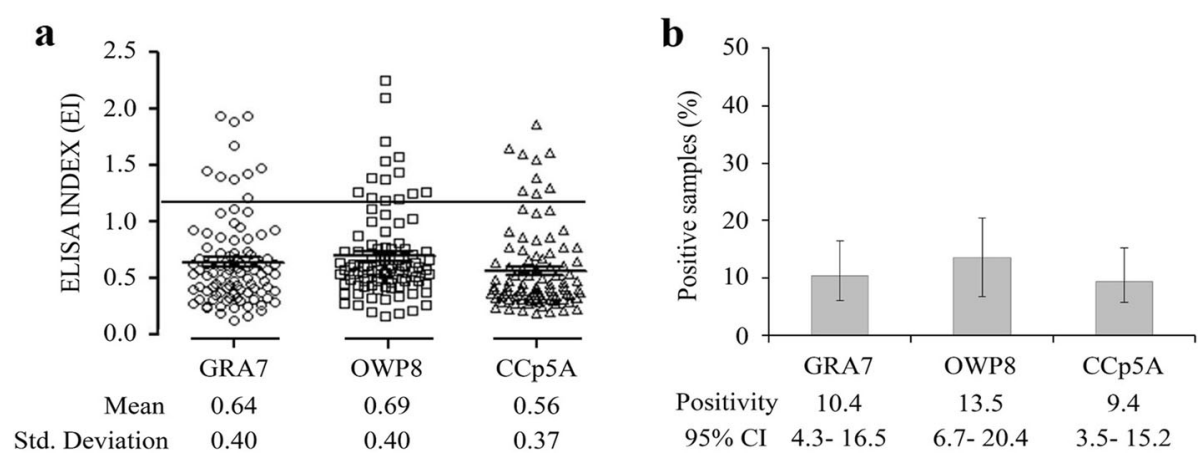

Fig. 4 Detection of anti-T. gondii antibodies in free-range chicken serum samples using different antigens. a The chicken serum samples were tested by GRA7-, OWP8-, and CCP5A-ELISAs, and the dashed line indicates the cut off of the reactions. b Positivity rates were evaluated using GRA7-, OWP8-, and CCP5A-ELISAs in serum samples from free-range chickens $(n=96)$ 

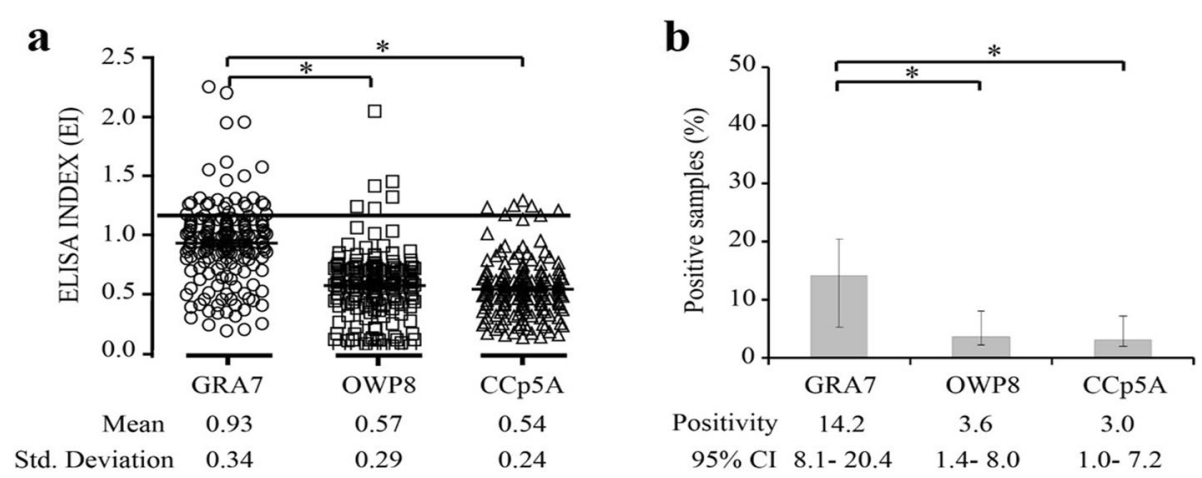

Fig. 5 Detection of anti-T. gondii antibodies in human serum samples by ELISAs using different antigens. a The human serum samples were tested by GRA7-, OWP8-, and CCP5A-ELISAs. The dashed line indicates the cut off of the reactions. b Positivity rates were evaluated using GRA7-, OWP8-, and CCP5A-ELISAs in human serum samples $(n=169) . * 0.05$

$75 \%$ cyst-driven infections were tested in humans in northeastern China [2, 20, 21].

Pigs and chickens are the major meat-producing animal species in China and other countries, and playing important role in the zoonotic transmission of T. gondii infection [21]. Free-range chickens have been considered as an important sentinel animal of soil contamination with $T$. gondii oocysts as they obtain food from the ground [22]. Thus, oocysts are considered the main infection form in chickens, as shown by a high oocyst-driven infection rates $(80-$ $100 \%)$ by different antigens. Though both OWP8 and $\mathrm{CCp} 5 \mathrm{~A}$ can be used as diagnostic markers for oocysts infection of $T$. gondii, their sensitivity may be different when applied to the clinical samples, which should be further evaluated using different animal species of $T$. gondii oocysts and cysts infections. On the other hand, it is possible for chickens to eat raw or undercooked meat containing $T$. gondii tissue cysts, though it is not the main route of infection. This study also showed pigs had a high oocystdriven infection rates, implying that pig feed are seriously contaminated with $T$. gondii oocysts in northeastern China.

\section{Conclusions}

The present study determined T. gondii oocyst-driven infection in pigs, chickens, and humans in Jilin Province, northeastern China. The differentiation of infective stages may help to design effective strategies to prevent $T$. gondii transmission in animals and humans in the studied areas.

\section{Methods}

\section{Serum samples}

Human blood samples were obtained from individuals who went to hospital for medical examination in Hun Chun City in Jilin Province. Animal blood samples were collected from the precaval vein of pigs and wing vein of chickens after permission was obtained from their owners in Changchun, Jilin Province, northeastern China in September, 2016. These animals were free-range in small peasant households. Sera were separated by centrifugation at $1500 \times \mathrm{g}$ for $5 \mathrm{~min}$ and stored at $-80^{\circ} \mathrm{C}$ until use. The T. gondii tachyzoite positive and negative porcine serum samples were kindly provided by Professor Xing-Quan Zhu at Lanzhou Veterinary Research Institute, Chinese Academy of Agricultural Sciences.

\section{Preparation of recombinant proteins GRA7, OWP8 and CCp5A}

Recombinant granule antigen protein GRA7 was prepared as described elsewhere [17]. The recombinant antigens OWP8 (http://toxodb.org/toxo/; Gene ID: TGVEG_271580) and CCp5A (GenBank ${ }^{\odot}$ accession number: EF517499) were expressed in Escherichia coli and purified using the standard techniques [13]. Briefly, the protein-encoding genes were cloned into pET-30a to generate recombinant plasmids pET30-OWP8 and pET30-CCp5A. The recombinant proteins were confirmed by restriction enzyme digestion and DNA sequencing analysis, and further processed for the expression of recombinant products in E. coli BL21 (DE3). The recombinant proteins were analyzed by SDSPAGE using a $12 \%$ polyacrylamide gel. The electrophoretic transfer of the recombinant proteins to a $0.2 \mu \mathrm{m}$ PVDF membrane was carried out by semi-dry transfer cell (Bio-Rad Trans-Blot ${ }^{\circ} \mathrm{SD}$ ) at $15 \mathrm{~V}$ for $35 \mathrm{~min}$. Blots of recombinant OWP8 and CCp5A were incubated with mouse anti-His tag sera as primary antibodies, followed by goat anti-mouse horseradish peroxidase conjugate, which were both diluted in 5\% skimmed milk TBST. The recombinant proteins were purified using the $\mathrm{Ni}$ NTA purification system (Qiagen, Hilden, Germany) according to the manufacturer's protocol. 
Enzyme-linked immunosorbent assays (ELISAs)

ELISA assays were conducted to evaluate anti- $T$. gondii antibodies in pigs, chickens and humans as described elsewhere with minor modification [17]. Briefly, microplates were coated with $50 \mu \mathrm{l}$ of GRA7 $(5 \mu \mathrm{g} / \mathrm{ml})$, OWP8 $(5 \mu \mathrm{g} / \mathrm{ml})$, or CCp5A $(10 \mu \mathrm{g} / \mathrm{ml})$, respectively. After overnight coating, the microplates were blocked with $100 \mu \mathrm{l}$ PBST-5\% skimmed milk for $1 \mathrm{~h}$. The pig, chicken, or human sera diluted at 1:50 with $1 \%$ skimmed milk PBST was added to each well, and incubated for $1 \mathrm{~h}$ at $37^{\circ} \mathrm{C}$, then $50 \mu \mathrm{l}$ horseradish peroxidase-conjugated anti-pig IgG, anti-chicken IgY, or anti-human IgG antibodies (Thermo Scientific, USA) diluted at 1:20,000 was added. After five washes, a TMB substrate solution was added and the reaction was stopped by $2 \mathrm{M} \mathrm{H}_{2} \mathrm{SO}_{4}$, and the optical density (OD) ELISA reaction was immediately measured by a microplate reader at $450 \mathrm{~nm}$. Each serum sample was performed in triplicate.

The cut off value of the reaction was calculated as the mean optical density (OD) of ten $T$. gondii -negative control sera plus 3 standard deviations. The antibody titer of each sample was expressed as the ELISA index (EI, EI=OD of each sample/cut off value) as described elsewhere [13]. Samples with EI values $\geq 1.2$ were considered positive.

\section{Western blot}

The GRA7-, OWP8-, and CCp5A-ELISAs results of chicken serum samples were verified by Western blot as previously described [17]. Briefly, the recombinant proteins were resolved in a $12 \%$ SDS-PAGE gel and transferred to PVDF membrane. After an overnight blocking TBST-5\% skimmed milk, the membranes were cut into strips and incubated with the serum samples. The membrane strips were further incubated with anti-pig IgG /anti-chicken IgY peroxidase-labeled conjugate antibodies (Thermo Scientific, USA) diluted at 1:5000. After washing, the strips were incubated with Super ECL Star (US Everbright ${ }^{\circ}$ Inc) and the protein bands were visualized the specific antibodies. A sample was considered positive if the protein bands were observed.

\section{Data analysis}

ELISA results were analyzed by the GraphPad Prism software version 5.0 (GraphPad Software, San Diego, CA, USA). Significant differences among IgG levels against recombinant proteins were determined by oneway ANOVA, while the differences among positivity rates were evaluated using Fisher's exact test. Values of $P<0.05$ were considered statistically significant.

\section{Abbreviations}

ANOVA: Analysis of variance; CCp5A: Limulus factor C-Coch-5b2-Lgl1 (LCCL) related protein; DNA: Deoxyribonucleic acid; El: ELISA index; ELISA: Enzymelinked immunosorbent assay; ERP: Embryogenesis- related protein;
GRA7: Granule antigen protein 7; OD: Optical density; OWP8: Oocyst wall protein 8; PVDF: Polyvinylidene fluoride; SDS-PAGE: Sodium dodecylsulfatepolyacrylamide gel electrophoresis

\section{Acknowledgements}

The authors would like to thank the animal owners and the individuals for participating in this study.

\section{Authors' contributions}

QL designed and supervised the study. QL and LXY wrote the original manuscript. ZDW, SEA, QL and LXY revised the manuscript. ZDW, SEA, QL and LXY performed the experiments. All authors have reviewed and approved the final manuscript.

\section{Funding}

This work was supported in part by the National Key R\&D Program of China (2017YFD0501304), the National Natural Science Foundation of China (31672542), and the Key Laboratory of Preventive Veterinary Medicine of Education Department in Guangdong Province of China (2014KTSPT037). The funders had no role in study design, in the collection, analysis and interpretation of data, in the writing the manuscript, or in the decision to submit the article for publication.

\section{Availability of data and materials}

The datasets used and/or analysed during the current study are available from the corresponding author on reasonable request.

\section{Ethics approval and consent to participate}

This study was approved by the Animal Ethics Committee (AMMS-MVIA2016-0023) and the Human Subject Committee (Approval No. AMMS-MVIH2016-002) of Military Veterinary Institute, Academy of Military Medical Sciences. We obtained the informed written consent from all animal owners and human participants. All animals used in this study were handled in accordance with the Guiding Principles of Animal Experiments by the Chinese Society of Laboratory Animal Sciences.

\section{Consent for publication}

Not applicable.

\section{Competing interests}

The authors declare that they have no competing interests.

Received: 3 February 2019 Accepted: 9 October 2019

Published online: 25 October 2019

\section{References}

1. Zhou P, Chen Z, Li HL, Zheng H, He S, Lin RQ, et al. Toxoplasma gondii infection in humans in China. Parasit Vectors. 2011:4:165.

2. Hill D, Dubey JP. Toxoplasma gondii: transmission, diagnosis and prevention. Clin Microbiol Infect. 2002;8:634-40.

3. Innes EA. A brief history and overview of Toxoplasma gondii. Zoonoses Public Health. 2010;57:1-7.

4. Dubey JP. Toxoplasma gondii infections in chickens (Gallus domesticus): prevalence, clinical disease, diagnosis and public health significance. Zoonoses Public Health. 2010;57:60-73.

5. Dubey JP, Edelhofer R, Marcet P, Vianna MC, Kwok OC, Lehmann T. Genetic and biologic characteristics of Toxoplasma gondii infections in free-range chickens from Austria. Vet Parasitol. 2005;133:299-306.

6. Dubey JP, Hotea I, Olariu TR, Jones JL, Darabus G. Epidemiological review of toxoplasmosis in humans and animals in Romania. Parasitology. 2014;141: $311-25$.

7. Dubey JP, Tiao N, Gebreyes WA, Jones JL. A review of toxoplasmosis in humans and animals in Ethiopia. Epidemiol Infect. 2012;140:1935-8.

8. Hoghooghi-Rad N, Afraa M. Prevalence of toxoplasmosis in humans and domestic animals in Ahwaz, capital of Khoozestan Province, south-West Iran. J Trop Med Hyg. 1993;96:163-8.

9. Holec-Gasior L, Drapala D, Dominiak-Gorski B, Kur J. Epidemiological study of Toxoplasma gondii infection among cattle in northern Poland. Ann Agric Environ Med. 2013;20:653-6. 
10. Jiang HH, Wang SC, Huang SY, Zhao L, Wang ZD, Zhu XQ, et al. Genetic characterization of Toxoplasma gondii isolates from pigs in Jilin Province, Northeastern China. Foodborne Pathog Dis. 2016;13:88-92.

11. Salman D, Okuda LH, Ueno A, Dautu G, Zhang F, Igarashi M. Evaluation of novel oocyst wall protein candidates of Toxoplasma gondii. Parasitol Int. 2017;66:643-51

12. Hill D, Coss C, Dubey JP, Wroblewski K, Sautter M, Hosten T, et al. Identification of a sporozoite-specific antigen from Toxoplasma gondii. J Parasitol. 2011;97:328-37.

13. Santana SS, Gebrim LC, Carvalho FR, Barros HS, Barros PC, Pajuaba AC, et al. CCp5A protein from Toxoplasma gondii as a serological marker of oocystdriven infections in humans and domestic animals. Front Microbiol. 2015;6:1305.

14. Jacobs D, Vercammen M, Saman E. Evaluation of recombinant dense granule antigen 7 (GRA7) of Toxoplasma gondii for detection of immunoglobulin $\mathrm{G}$ antibodies and analysis of a major antigenic domain. Clin Diagn Lab Immunol. 1999;6:24-9.

15. Pietkiewicz H, Hiszczynska-Sawicka E, Kur J, Petersen E, Nielsen HV, Paul M, et al. Usefulness of Toxoplasma gondii recombinant antigens (GRA1, GRA7 and SAG1) in an immunoglobulin G avidity test for the serodiagnosis of toxoplasmosis. Parasitol Res. 2007;100:333-7.

16. Sun X, Wang Z, Li J, Wei F, Liu Q. Evaluation of an indirect ELISA using recombinant granule antigen GRA1, GRA7 and soluble antigens for serodiagnosis of Toxoplasma gondii infection in chickens. Res Vet Sci. 2015;100:161-4.

17. Wang Z, Ge W, Li J, Song M, Sun H, Wei F, et al. Production and evaluation of recombinant granule antigen protein GRA7 for serodiagnosis of Toxoplasma gondii infection in cattle. Foodborne Pathog Dis. 2014;11:734-9.

18. Wang Z, Ge W, Huang SY, Li J, Zhu XQ, Liu Q. Evaluation of recombinant granule antigens GRA1 and GRA7 for serodiagnosis of Toxoplasma gondii infection in dogs. BMC Vet Res. 2014;10:158.

19. Cai Y, Wang Z, Li J, Li N, Wei F, Liu Q. Evaluation of an indirect ELISA using recombinant granule antigen GRA7 for serodiagnosis of Toxoplasma gondii infection in cats. J Parasitol. 2015;101:37-40.

20. Dubey JP. Toxoplasmosis - a waterborne zoonosis. Vet Parasitol. 2004;126: 57-72.

21. Schluter D, Daubener W, Schares G, Groß U, Pleyer U, Luder C. Animals are key to human toxoplasmosis. Int J Med Microbiol. 2014;304:917-29.

22. Zhao G, Shen B, Xie Q, Xu LX, Yan RF, Song XK, et al. Detection of Toxoplasma gondii in free-range chickens in China based on circulating antigens and antibodies. Vet Parasitol. 2012;185:72-7.

\section{Publisher's Note}

Springer Nature remains neutral with regard to jurisdictional claims in published maps and institutional affiliations.

Ready to submit your research? Choose BMC and benefit from:

- fast, convenient online submission

- thorough peer review by experienced researchers in your field

- rapid publication on acceptance

- support for research data, including large and complex data types

- gold Open Access which fosters wider collaboration and increased citations

- maximum visibility for your research: over $100 \mathrm{M}$ website views per year

At $\mathrm{BMC}$, research is always in progress.

Learn more biomedcentral.com/submissions 